International Journal of Pure and Applied Mathematics

Volume 92 No. 5 2014, 719-729

ISSN: 1311-8080 (printed version); ISSN: 1314-3395 (on-line version)

url: http://www.ijpam.eu

doi: http://dx.doi.org/10.12732/ijpam.v92i5.7

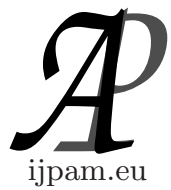

\title{
UPPER APPROXIMATION OPERATORS INDUCED BY ALEXANDROV FUZZY TOPOLOGIES
}

\author{
Yong Chan Kim \\ Department of Mathematics \\ Gangneung-Wonju University \\ Gangneung, Gangwondo, 210-702, KOREA
}

\begin{abstract}
In this paper, we investigate the properties of upper approximation operators induced by Alexandrov fuzzy topologies in complete residuated lattices. We give their examples.
\end{abstract}

AMS Subject Classification: 03E72, 03G10, 06A15, 06F07

Key Words: complete residuated lattices, fuzzy preorders, join preserving maps, upper approximation operators, Alexandrov fuzzy topologies

\section{Introduction}

Höhle [3] introduced $L$-fuzzy topologies and $L$-fuzzy interior operators. The relationship between rough set theory and topological spaces was investigated in sets [7]. Hájek [2] introduced a complete residuated lattice which is an algebraic structure for many valued logic. It is an important mathematical tool for algebraic structure of fuzzy contexts [1-6, 8,9, 12-15]. Kim [5,6] investigated the properties of join (resp. meet, meet join, join meet) preserving operators in complete residuated lattices.

In this paper, we investigate the properties of upper approximation operators induced by Alexandrov fuzzy topologies in complete residuated lattices in a sense as Höhle's $L$-fuzzy topologies and $L$-fuzzy interior operators [3]. We give their examples.

Received: March 1, 2014

(c) 2014 Academic Publications, Ltd. url: www.acadpubl.eu 


\section{Preliminaries}

Definition 2.1. [1-3] A structure $(L, \vee, \wedge, \odot, \rightarrow, \perp, \top)$ is called a complete residuated lattice iff it satisfies the following properties:

(L1) $(L, \vee, \wedge, \perp, \top)$ is a complete lattice where $\perp$ is the bottom element and $\top$ is the top element;

(L2) $(L, \odot, \top)$ is a monoid;

(L3) It has an adjointness,i.e.

$$
x \leq y \rightarrow z \text { iff } x \odot y \leq z .
$$

An operator ${ }^{*}: L \rightarrow L$ defined by $a^{*}=a \rightarrow \perp$ is called strong negations if $a^{* *}=a$.

$$
\top_{x}(y)=\left\{\begin{array}{ll}
\top, & \text { if } y=x, \\
\perp, & \text { otherwise. }
\end{array} \top_{x}^{*}(y)= \begin{cases}\perp, & \text { if } y=x, \\
\top, & \text { otherwise. }\end{cases}\right.
$$

In this paper, we assume that $\left(L, \vee, \wedge, \odot, \rightarrow,{ }^{*}, \perp, \top\right)$ be a complete residuated lattice with a strong negation *.

Definition 2.2. $[8,9]$ Let $X$ be a set. A function $e_{X}: X \times X \rightarrow L$ is called:

(E1) reflexive if $e_{X}(x, x)=1$ for all $x \in X$,

(E2) transitive if $e_{X}(x, y) \odot e_{X}(y, z) \leq e_{X}(x, z)$, for all $x, y, z \in X$,

(E3) if $e_{X}(x, y)=e_{X}(y, x)=1$, then $x=y$.

If $e$ satisfies (E1) and (E2), $\left(X, e_{X}\right)$ is a fuzzy preorder set. If $e$ satisfies (E1), (E2) and (E3), $\left(X, e_{X}\right)$ is a fuzzy partially order set (simply, fuzzy poset).

Example 2.3. (1) We define a function $e_{L}: L \times L \rightarrow L$ as $e_{L}(x, y)=x \rightarrow y$. Then $\left(L, e_{L}\right)$ is a fuzzy poset.

(2) We define a function $e_{L^{X}}: L^{X} \times L^{X} \rightarrow L$ as $e_{L^{X}}(A, B)=\bigwedge_{x \in X}(A(x) \rightarrow$ $B(x))$. Then $\left(L^{X}, e_{L^{X}}\right)$ is a fuzzy poset from Lemma $2.4(9)$.

Lemma 2.4. $[1,2]$ Let $\left(L, \vee, \wedge, \odot, \rightarrow,^{*}, \perp, \top\right)$ be a complete residuated lattice with a strong negation ${ }^{*}$. For each $x, y, z, x_{i}, y_{i} \in L$, the following properties hold.

(1) If $y \leq z$, then $x \odot y \leq x \odot z$.

(2) If $y \leq z$, then $x \rightarrow y \leq x \rightarrow z$ and $z \rightarrow x \leq y \rightarrow x$.

(3) $x \rightarrow y=\top$ iff $x \leq y$.

(4) $x \rightarrow \top=\top$ and $\top \rightarrow x=x$.

(5) $x \odot y \leq x \wedge y$.

(6) $x \odot\left(\bigvee_{i \in \Gamma} y_{i}\right)=\bigvee_{i \in \Gamma}\left(x \odot y_{i}\right)$ and $\left(\bigvee_{i \in \Gamma} x_{i}\right) \odot y=\bigvee_{i \in \Gamma}\left(x_{i} \odot y\right)$. 
(7) $x \rightarrow\left(\bigwedge_{i \in \Gamma} y_{i}\right)=\bigwedge_{i \in \Gamma}\left(x \rightarrow y_{i}\right)$ and $\left(\bigvee_{i \in \Gamma} x_{i}\right) \rightarrow y=\bigwedge_{i \in \Gamma}\left(x_{i} \rightarrow y\right)$. $\left.y_{i}\right)$.

(8) $\bigvee_{i \in \Gamma} x_{i} \rightarrow \bigvee_{i \in \Gamma} y_{i} \geq \bigwedge_{i \in \Gamma}\left(x_{i} \rightarrow y_{i}\right)$ and $\bigwedge_{i \in \Gamma} x_{i} \rightarrow \bigwedge_{i \in \Gamma} y_{i} \geq \bigwedge_{i \in \Gamma}\left(x_{i} \rightarrow\right.$

(9) $(x \rightarrow y) \odot x \leq y$ and $(y \rightarrow z) \odot(x \rightarrow y) \leq(x \rightarrow z)$.

(10) $x \rightarrow y \leq(y \rightarrow z) \rightarrow(x \rightarrow z)$ and $x \rightarrow y \leq(z \rightarrow x) \rightarrow(z \rightarrow y)$.

(11) $\bigwedge_{i \in \Gamma} x_{i}^{*}=\left(\bigvee_{i \in \Gamma} x_{i}\right)^{*}$ and $\bigvee_{i \in \Gamma} x_{i}^{*}=\left(\bigwedge_{i \in \Gamma} x_{i}\right)^{*}$.

(12) $(x \odot y) \rightarrow z=x \rightarrow(y \rightarrow z)=y \rightarrow(x \rightarrow z)$ and $(x \odot y)^{*}=x \rightarrow y^{*}$.

(13) $x^{*} \rightarrow y^{*}=y \rightarrow x$ and $(x \rightarrow y)^{*}=x \odot y^{*}$.

(14) $y \rightarrow z \leq x \odot y \rightarrow x \odot z$.

Definition 2.5. [5,6] A map $\mathcal{H}: L^{X} \rightarrow L^{Y}$ is called a join preserving map if it satisfies the following conditions, for all $A, A_{i} \in L^{X}$, and $\alpha \in L$,

(J1) $\mathcal{H}(\alpha \odot A)=\alpha \odot \mathcal{H}(A)$,

(J2) $\mathcal{H}\left(\bigvee_{i \in I} A_{i}\right)=\bigvee_{i \in I} \mathcal{H}\left(A_{i}\right)$.

A join preserving operator $\mathcal{H}: L^{X} \rightarrow L^{X}$ is called an upper approximation operator iff it satisfies the following conditions

(H1) $A \leq \mathcal{H}(A)$,

(H2) $\mathcal{H}(\mathcal{H}(A)) \leq \mathcal{H}(A)$, for all $A \in L^{X}$.

Definition 2.6. [6] An operator $\mathbf{T}: L^{X} \rightarrow L$ is called an Alexandrov fuzzy topology on $X$ iff it satisfies the following conditions:

(T1) $\mathbf{T}(\alpha)=\top$,

(T2) $\mathbf{T}\left(\bigwedge_{i \in \Gamma} A_{i}\right) \geq \bigwedge_{i \in \Gamma} \mathbf{T}\left(A_{i}\right)$ and $\mathbf{T}\left(\bigvee_{i \in \Gamma} A_{i}\right) \geq \bigwedge_{i \in \Gamma} \mathbf{T}\left(A_{i}\right)$,

(T3) $\mathbf{T}(\alpha \odot A) \geq \mathbf{T}(A)$,

(T4) $\mathbf{T}(\alpha \rightarrow A) \geq \mathbf{T}(A)$.

Theorem 2.7. [6] Let $\mathbf{T}: L^{X} \rightarrow L$ be an Alexandrov fuzzy topology. Define $\mathbf{T}^{*}(A)=\mathbf{T}\left(A^{*}\right)$. Then $\mathbf{T}^{*}$ is an Alexandrov fuzzy topology.

Theorem 2.8. [6] Let $\mathcal{H}$ be a join preserving map. Define $\mathbf{T}_{H}: L^{X} \rightarrow L$ as

$$
\mathbf{T}_{H}(A)=\bigwedge_{x \in X}(\mathcal{H}(A)(x) \rightarrow A(x))=e_{L^{X}}(\mathcal{H}(A), A) .
$$

Then we have the following properties.

(1) $\mathbf{T}_{H}$ is an Alexandrov fuzzy topology on $X$.

(2) $\mathbf{T}_{H}(A)=\bigwedge_{x, y \in X}\left(\mathcal{H}\left(\top_{x}\right)(y) \rightarrow(A(x) \rightarrow A(y))\right.$ such that $\mathbf{T}_{H}(A) \geq$ $\bigwedge_{x \neq y \in X} \mathcal{H}\left(\top_{x}\right)(y)$.

(3) If $\mathcal{H}$ is an upper approximation operator, then $\mathbf{T}_{H}\left(\mathcal{H}\left(\top_{x}\right)\right)=T$. 
(4) If $\mathcal{H}^{-1}$ is a join preserving map such that $\mathcal{H}^{-1}\left(\top_{x}\right)(y)=\mathcal{H}\left(\top_{y}\right)(x)$ for all $x, y \in X$. Define $\mathbf{T}_{H}^{*}(A)=\mathbf{T}_{H}\left(A^{*}\right)$. Then $\mathbf{T}_{H}^{*}=\mathbf{T}_{H^{-1}}$ is an Alexandrov fuzzy topology.

(5) If $\mathcal{H}$ is an upper approximation operator, then $\mathcal{H}^{-1}$ is an upper approximation operator such that

$$
\mathbf{T}_{H}\left(\mathcal{H}^{-1 *}\left(\top_{x}\right)\right)=\mathbf{T}_{H^{-1}}\left(\mathcal{H}^{*}\left(\top_{x}\right)\right)=\top .
$$

\section{Upper Approximation Operators Induced by Alexandrov Fuzzy Topologies}

Theorem 3.1. Let $\mathbf{T}$ be an Alexandrov fuzzy topology on $X$. Define $\mathcal{H}_{T}$ : $L^{X} \times L \rightarrow L^{X}$ as follows

$$
\mathcal{H}_{T}(A, r)=\bigwedge\left\{B \in L^{X} \mid A \leq B, \mathbf{T}(B) \geq r^{*}\right\}
$$

Then we have the following properties.

(1) $\mathcal{H}_{T}(-, r): L^{X} \rightarrow L^{X}$ is an upper approximation operator.

(2) If $r \leq s$, then $\mathcal{H}_{T}(A, s) \leq \mathcal{H}_{T}(A, r)$ for all $A \in L^{X}$.

(3) There exists a preorder $R_{T}^{r} \in L^{X \times X}$ such that

$$
\mathcal{H}_{T}(A, r)=\bigvee_{x \in X}\left(A(x) \odot R_{T}^{r}(x, y)\right) .
$$

(4) If $r \leq s$, then $R_{T}^{r} \geq R_{T}^{s}$ for all $A \in L^{X}$.

(5) If $\mathcal{H}_{T}\left(A, r_{i}\right)=B$ for all $i \in \Gamma \neq \emptyset$, then $\mathcal{H}_{T}\left(A, \bigwedge_{i \in \Gamma} r_{i}\right)=B$.

(6) Define $\mathbf{T}_{H_{T}}: L^{X} \rightarrow L$ as

$$
\mathbf{T}_{H_{T}}(A)=\bigvee\left\{r_{i}^{*} \in L \mid \mathcal{H}_{T}\left(A, r_{i}\right)=A\right\}
$$

Then $\mathbf{T}_{H_{T}}=\mathbf{T}$ is an Alexandrov fuzzy topology on $X$.

(7) There exists an Alexandrov fuzzy topology $\mathbf{T}^{r}$ such that

$$
\mathbf{T}^{r}(A)=e_{L^{X}}\left(\mathcal{H}_{T}(A, r), A\right) .
$$

(8) If $r \leq s$, then $\mathbf{T}^{r} \leq \mathbf{T}^{s}$ for all $A \in L^{X}$.

(9) Define $\mathbf{T}_{T}: L^{X} \rightarrow L$ as

$$
\mathbf{T}_{T}(A)=\bigvee\left\{r^{*} \in L \mid \mathbf{T}^{r}(A)=\top\right\}
$$


Then $\mathbf{T}_{T}=\mathbf{T}=\mathbf{T}_{H_{T}}$ is an Alexandrov fuzzy topology on $X$.

Proof. (1) First, $\mathcal{H}_{T}(-, r)$ is a join preserving map from the following statements. Since $\alpha \odot \bigwedge(\alpha \rightarrow B) \leq \bigwedge(\alpha \odot(\alpha \rightarrow B)) \leq \bigwedge B$, we have

$$
\begin{aligned}
& \mathcal{H}_{T}(\alpha \odot A, r)=\bigwedge\left\{B \in L^{X} \mid \alpha \odot A \leq B, \mathbf{T}(B) \geq r^{*}\right\} \\
& \geq \alpha \odot \bigwedge\left\{\alpha \rightarrow B \in L^{X} \mid A \leq \alpha \rightarrow B, \mathbf{T}(\alpha \rightarrow B) \geq r^{*}\right\} \\
& \geq \alpha \odot \bigwedge\left\{C \in L^{X} \mid A \leq C, \mathbf{T}(C) \geq r^{*}\right\}=\alpha \odot \mathcal{H}_{T}(A, r) .
\end{aligned}
$$

Since $\mathbf{T}\left(\mathcal{H}_{T}(A, r)\right) \geq r^{*}$ and $\alpha \odot A \leq \alpha \odot \mathcal{H}_{T}(A, r)$, then $\mathbf{T}\left(\alpha \odot \mathcal{H}_{T}(A, r)\right) \geq r^{*}$ and $\mathcal{H}_{T}(\alpha \odot A, r) \leq \alpha \odot \mathcal{H}_{T}(A, r)$. Hence $\mathcal{H}_{T}(\alpha \odot A, r) \leq \alpha \odot \mathcal{H}_{T}(A, r)$.

By the definition of $\mathcal{H}_{T}(-, r), \bigvee_{i \in \Gamma} \mathcal{H}_{T}\left(A_{i}, r\right) \leq \mathcal{H}_{T}\left(\bigvee_{i \in \Gamma} A_{i}, r\right)$

Since $\bigvee_{i \in \Gamma} A_{i} \leq \bigvee_{i \in \Gamma} \mathcal{H}_{T}\left(A_{i}, r\right)$ and $\mathbf{T}\left(\bigvee_{i \in \Gamma} \mathcal{H}_{T}\left(A_{i}, r\right)\right) \geq r^{*}$, then

$$
\mathcal{H}_{T}\left(\bigvee_{i \in \Gamma} A_{i}, r\right) \leq \bigvee_{i \in \Gamma} \mathcal{H}_{T}\left(A_{i}, r\right)
$$

(H1) By the definition of $\mathcal{H}_{T}(-, r), A \leq \mathcal{H}_{T}(A, r)$.

(H2) By $(\mathrm{H} 1), \mathcal{H}_{T}(A, r) \leq \mathcal{H}_{T}\left(\mathcal{H}_{T}(A, r), r\right)$.

Since $\mathcal{H}_{T}\left(\mathcal{H}_{T}(A, r), r\right)=\bigwedge\left\{B \in L^{X} \mid \mathcal{H}_{T}(A, r) \leq B, \mathbf{T}(B) \geq r^{*}\right\}$ and $\mathbf{T}\left(\mathcal{H}_{T}(A, r)\right) \geq r^{*}$, then $\mathcal{H}_{T}\left(\mathcal{H}_{T}(A, r), r\right)=\mathcal{H}_{T}(A, r)$. Hence $\mathcal{H}_{T}(-, r)$ is an upper approximation operator.

(2) For $r \leq s$, since $\mathbf{T}(B) \geq r^{*} \geq s^{*}$, we have $\mathcal{H}_{T}(A, s) \leq \mathcal{H}_{T}(A, r)$ for all $A \in L^{X}$.

(3) Put $R_{T}^{r}(x, y)=\mathcal{H}_{T}\left(\top_{x}, r\right)(y)$. Since $A=\bigvee_{x \in X}\left(A(x) \odot \top_{x}\right)$ and $\mathcal{H}_{T}$ is a join preserving map, we have

$$
\begin{aligned}
& \mathcal{H}_{T}(A, r)(y)=\mathcal{H}_{T}\left(\bigvee_{x \in X}\left(A(x) \odot \top_{x}\right), r\right)(y) \\
& =\bigvee_{x \in X}\left(A(x) \odot \mathcal{H}_{T}\left(\top_{x}, r\right)(y)\right)=\bigvee_{x \in X}\left(A(x) \odot R_{T}^{r}(x, y)\right)
\end{aligned}
$$

By $(\mathrm{H} 1), R_{T}^{r}(x, x)=\mathcal{H}_{T}\left(\top_{x}, r\right)(x) \geq \top_{x}(x)=\top$.

$$
\begin{aligned}
& \bigvee_{y \in X}\left(R_{T}^{r}(x, y) \odot R_{T}^{r}(y, z)\right) \\
& =\bigvee_{y \in X}\left(\mathcal{H}_{T}\left(\top_{x}, r\right)(y) \odot \mathcal{H}_{T}\left(\top_{y}, r\right)(z)\right) \\
& \left.=\mathcal{H}_{T}\left(\bigvee_{y \in X}\left(\mathcal{H}_{T}\left(\top_{x}, r\right)(y) \odot \top_{y}\right), r\right)(z)\right) \\
& =\mathcal{H}_{T}\left(\mathcal{H}_{T}\left(\top_{x}, r\right), r\right)(z) \leq \mathcal{H}_{T}\left(\top_{x}, r\right)(z)=R_{T}^{r}(x, z)
\end{aligned}
$$

Hence $R_{T}^{r}$ is a fuzzy preorder.

(4) If $r \leq s$, by $(2), R_{T}^{r}(x, y)=\mathcal{H}_{T}\left(\top_{x}, r\right)(y) \geq R_{T}^{s}(x, y)=\mathcal{H}_{T}\left(\top_{x}, s\right)(y)$.

(5) Let $\mathcal{H}_{T}\left(A, r_{i}\right)=B$ for all $i \in \Gamma \neq \emptyset$. Since $\mathbf{T}(B)=\mathbf{T}\left(\mathcal{H}_{T}\left(A, r_{i}\right)\right) \geq r_{i}^{*}$, then $\mathbf{T}(B) \geq \bigvee_{i \in \Gamma} r_{i}^{*}=\left(\bigwedge_{i \in \Gamma} r_{i}\right)^{*}$. Hence

$$
B=\mathcal{H}_{T}\left(B, \bigwedge_{i \in \Gamma} r_{i}\right) \geq \mathcal{H}_{T}\left(A, \bigwedge_{i \in \Gamma} r_{i}\right)
$$


By $(2), \mathcal{H}_{T}\left(A, \bigwedge_{i \in \Gamma} r_{i}\right) \geq \mathcal{H}_{T}\left(A, r_{i}\right)=B$. Thus $\mathcal{H}_{T}\left(A, \bigwedge_{i \in \Gamma} r_{i}\right)=B$.

(6) Since $\mathbf{T}(A)=\mathbf{T}\left(\mathcal{H}_{T}\left(A, r_{i}\right)\right) \geq r_{i}^{*}$, for $\mathcal{H}_{T}\left(A, r_{i}\right)=A$,

$$
\mathbf{T}_{H_{T}}(A)=\bigvee\left\{r_{i}^{*} \in L \mid \mathcal{H}_{T}\left(A, r_{i}\right)=A\right\} \leq \mathbf{T}(A) .
$$

Since $\mathcal{H}_{T}\left(A, \mathbf{T}^{*}(A)\right)=A$,

$$
\mathbf{T}_{H_{T}}(A)=\bigvee\left\{r_{i}^{*} \in L \mid \mathcal{H}_{T}\left(A, r_{i}\right)=A\right\} \geq \mathbf{T}(A) .
$$

Hence $\mathbf{T}_{H_{T}}=\mathbf{T}$.

(7) By (1), since $\mathcal{H}_{T}(-, r)$ is an upper approximation operator, by Theorem 2.8, there exists an Alexandrov fuzzy topology $\mathbf{T}^{r}$ such that

$$
\mathbf{T}^{r}(A)=e_{L^{X}}\left(\mathcal{H}_{T}(A, r), A\right) .
$$

(8) Since $\mathcal{H}_{T}(A, s) \leq \mathcal{H}_{T}(A, r)$ for $r \leq s$, $\mathbf{T}^{s}(A)=e_{L^{x}}\left(\mathcal{H}_{T}(A, s), A\right) \geq$ $e_{L^{X}}\left(\mathcal{H}_{T}(A, r), A\right)=\mathbf{T}^{r}(A)$.

(9) Since $\mathbf{T}^{r}(A)=e_{L^{X}}\left(\mathcal{H}_{T}(A, r), A\right)=\top$ iff $A=\mathcal{H}_{T}(A, r)$, by (6), the result holds.

Corollary 3.2. Let $\mathbf{T}$ be an Alexandrov fuzzy topology on $X$. Define $\mathcal{H}_{T^{*}}: L^{X} \times L \rightarrow L^{X}$ as follows

$$
\mathcal{H}_{T^{*}}(A, r)=\bigwedge\left\{B \in L^{X} \mid A \leq B, \mathbf{T}^{*}(B)=\mathbf{T}\left(B^{*}\right) \geq r^{*}\right\}
$$

Then we have the following properties.

(1) $\mathcal{H}_{T^{*}}(-, r): L^{X} \rightarrow L^{X}$ is an upper approximation operator.

(2) If $r \leq s$, then $\mathcal{H}_{T^{*}}(A, s) \leq \mathcal{H}_{T^{*}}(A, r)$ for all $A \in L^{X}$.

(3) There exists a preorder $R_{T^{*}}^{r} \in L^{X \times X}$ such that

$$
\mathcal{H}_{T^{*}}(A, r)=\bigvee_{x \in X}\left(A(x) \odot R_{T^{*}}^{r}(x, y)\right) .
$$

(4) If $r \leq s$, then $R_{T^{*}}^{r} \geq R_{T^{*}}^{s}$ for all $A \in L^{X}$.

(5) If $\mathcal{H}_{T^{*}}\left(A, r_{i}\right)=B$ for all $i \in \Gamma \neq \emptyset$, then $\mathcal{H}_{T^{*}}\left(A, \bigwedge_{i \in \Gamma} r_{i}\right)=B$.

(6) Define $\mathbf{T}_{H_{T^{*}}}: L^{X} \rightarrow L$ as

$$
\mathbf{T}_{H_{T^{*}}}(A)=\bigvee\left\{r_{i}^{*} \in L \mid \mathcal{H}_{T^{*}}\left(A, r_{i}\right)=A\right\}
$$

Then $\mathbf{T}_{H_{T^{*}}}=\mathbf{T}^{*}$ is an Alexandrov fuzzy topology on $X$.

(7) There exists an Alexandrov fuzzy topology $\mathbf{T}^{* r}$ such that

$$
\mathbf{T}^{* r}(A)=e_{L^{X}}\left(\mathcal{H}_{T^{*}}(A, r), A\right) .
$$


(8) If $r \leq s$, then $\mathbf{T}^{* r} \leq \mathbf{T}^{* s}$ for all $A \in L^{X}$.

(9) Define $\mathbf{T}_{T^{*}}: L^{X} \rightarrow L$ as

$$
\mathbf{T}_{T^{*}}(A)=\bigvee\left\{r^{*} \in L \mid \mathbf{T}^{* r}(A)=\top\right\} .
$$

Then $\mathbf{T}_{T^{*}}=\mathbf{T}^{*}=\mathbf{T}_{H_{T^{*}}}$ is an Alexandrov fuzzy topology on $X$.

Example 3.3. Let $\mathbf{T}$ be an Alexandrov fuzzy topology on $X$ as follows

$$
\mathbf{T}(A)= \begin{cases}1, & \text { if } A=\bar{\alpha} \\ 0.4, & \text { otherwise. }\end{cases}
$$

(1) $\mathcal{H}_{T}(-, r): L^{X} \rightarrow L^{X}$ is an upper approximation operator as follows

$$
\mathcal{H}_{T}(A, r)= \begin{cases}A, & \text { if } 0.6 \leq r \leq 1, \\ \bar{\alpha}, \alpha=\bigvee_{x \in X} A(x), & \text { if } 0 \leq r<0.6\end{cases}
$$

(2) $\mathcal{H}_{T}(A, r)=\bigvee_{x \in X}\left(R_{T}^{r}(x, y) \odot A(x)\right)$ with $R_{T}^{r} \in L^{X \times X}$ as follows

$$
R_{T}^{r}(x, y)=\mathcal{H}_{T}\left(1_{x}, r\right)(y)= \begin{cases}1_{x}(y), & \text { if } 0.6 \leq r \leq 1, \\ \overline{1}, 1=\bigvee_{z \in X} 1_{x}(z), & \text { if } 0 \leq r<0.6\end{cases}
$$

(3) $\mathbf{T}^{r}$ is an Alexandrov fuzzy topology on $X$ as follows

$$
\mathbf{T}^{r}(A)= \begin{cases}\mathbf{T}_{1}(A), & \text { if } 0.6 \leq r \leq 1 \\ \mathbf{T}_{2}(A), & \text { if } 0 \leq r<0.6\end{cases}
$$

where

$$
\mathbf{T}_{1}(A)=1, \forall A \in L^{X}, \quad \mathbf{T}_{2}(A)= \begin{cases}1, & \text { if } A=\bar{\alpha}, \\ 0, & \text { otherwise. }\end{cases}
$$

Moreover, $\mathbf{T}_{T}(A)=\bigvee\left\{r^{*} \in L \mid \mathbf{T}^{r}(A)=1\right\}=\mathbf{T}_{M_{T}}(A)=\mathbf{T}(A)=\mathbf{T}^{*}(A)$ for all $A \in L^{X}$.

Example 3.4. Let $\left(L=[0,1], \odot, \rightarrow,^{*}\right)$ be a complete residuated lattice with a strong negation defined by

$$
a \odot b=(a+b-1) \vee 0, a \rightarrow b=(1-a+b) \wedge 1, a^{*}=1-a .
$$

(1) Let $X=\{x, y, z\}$ be a set. Define a map $\mathbf{T}:[0,1]^{X} \rightarrow[0,1]$ as

$$
\mathbf{T}(A)=(1-A(x)+A(z)) \wedge 1=A(x) \rightarrow A(z) .
$$

Trivially, $\mathbf{T}(\bar{\alpha})=\alpha$ 
Since $\alpha \odot A(x) \rightarrow \alpha \odot A(z) \geq A(x) \rightarrow A(z), \mathbf{T}(\alpha \odot A) \geq \mathbf{T}(A)$. Since $(\alpha \rightarrow A(x)) \rightarrow(\alpha \rightarrow A(z)) \geq A(x) \rightarrow A(z), \mathbf{T}(\alpha \rightarrow A) \geq \mathbf{T}(A)$. By Lemma $2.4(8), \mathbf{T}\left(\bigvee_{i \in \Gamma} A_{i}\right) \geq \bigwedge_{i \in \Gamma} \mathbf{T}\left(A_{i}\right)$ and $\mathbf{T}\left(\bigwedge_{i \in \Gamma} A_{i}\right) \geq \bigwedge_{i \in \Gamma} \mathbf{T}\left(A_{i}\right)$. Hence $\mathbf{T}$ is an Alexandrov fuzzy topology.

By Theorem 2.8(1), we obtain an upper approximation operator $\mathcal{H}_{T}(-, r)$ : $L^{X} \rightarrow L^{X}$ as follows:

$$
\mathcal{H}_{T}\left(1_{x}, r\right)(z)=\bigwedge\left\{B(z) \mid B \geq 1_{x}, \mathbf{T}(B) \geq r^{*}\right\}
$$

Since $B(x)=1$ and $\mathbf{T}(B)=1-1+B(z) \geq 1-r$, then $B(z) \geq 1-r$. We have $\mathcal{H}_{T}\left(1_{x}, r\right)(z)=1-r$.

$$
\begin{gathered}
\mathcal{H}_{T}\left(1_{x}, r\right)(x)=\bigwedge\left\{B(x) \mid B \geq 1_{x}, \mathbf{T}(B) \geq r^{*}\right\}=1 \\
\mathcal{H}_{T}\left(1_{x}, r\right)(y)=\bigwedge\left\{B(y) \mid B \geq 1_{x}, \mathbf{T}(B) \geq r^{*}\right\}=0 \\
\mathcal{H}_{T}\left(1_{z}, r\right)(x)=\bigwedge\left\{B(x) \mid B \geq 1_{z}, \mathbf{T}(B) \geq r^{*}\right\}
\end{gathered}
$$

Since $B(z)=1$ and $\mathbf{T}(B)=(1-B(x)+1) \wedge 1=1$, then $\mathcal{H}_{T}\left(1_{z}, r\right)(x)=0$.

$$
\left(\begin{array}{ccc}
\mathcal{H}_{T}\left(1_{x}, r\right)(x)=1 & \mathcal{H}_{T}\left(1_{x}, r\right)(y)=0 & \mathcal{H}_{T}\left(1_{x}, r\right)(z)=1-r \\
\mathcal{H}_{T}\left(1_{y}, r\right)(x)=0 & \mathcal{H}_{T}\left(1_{y}, r\right)(y)=1 & \mathcal{H}_{T}\left(1_{y}, r\right)(z)=0 \\
\mathcal{H}_{T}\left(1_{z}, r\right)(x)=0 & \mathcal{H}_{T}\left(1_{z}, r\right)(y)=0 & \mathcal{H}_{T}\left(1_{z}, r\right)(z)=1
\end{array}\right)
$$

For $A=\bigvee_{x \in X}\left(A(x) \odot \top_{x}\right)$, we have

$$
\begin{aligned}
& \mathcal{H}_{T}(A, r)(y)=\bigvee_{x \in X}\left(A(x) \odot \mathcal{H}_{T}\left(\top_{x}, r\right)(y)\right) \\
& \mathcal{H}_{T}(A, r)=(A(x), A(y), A(z) \vee(A(x)-r))
\end{aligned}
$$

If $A(x)-r \leq A(z)$, then $\mathcal{H}_{T}(A, r)=A$. Thus

$$
\begin{aligned}
\mathbf{T}_{H_{T}}(A) & =\bigvee\left\{r^{*} \in L \mid \mathcal{H}_{T}(A, r)=A\right\} \\
& =(1-A(x)+A(z)) \wedge 1=\mathbf{T}(A) .
\end{aligned}
$$

Moreover, we obtain

$$
\begin{aligned}
\mathbf{T}^{r}(A) & =\bigwedge_{x \in X}\left(\mathcal{H}_{T}(A, r)(x) \rightarrow A(x)\right) \\
& =A(z) \vee(A(x)-r) \rightarrow A(z) \\
& =(A(z) \rightarrow A(z)) \wedge((A(x)-r) \rightarrow A(z)) \\
& =(1+r-A(x)+A(z)) \vee 0 .
\end{aligned}
$$




$$
\begin{aligned}
\mathbf{T}_{T}(A) & =\bigvee\left\{1-r \in L \mid \mathbf{T}^{r}(A)=1\right\} \\
& =(1-A(x)+A(z)) \wedge 1
\end{aligned}
$$

Hence $\mathbf{T}_{T}=\mathbf{T}_{H_{T}}=\mathbf{T}$. with

Since $R_{T}^{r}(x, y)=\mathcal{H}_{T}\left(1_{x}, r\right)(y)$, then $\mathcal{H}_{T}(A, r)(y)=\bigvee_{x \in X}\left(A(x) \odot R_{T}^{r}(x, y)\right)$

$$
R_{T}^{r}=\left(\begin{array}{ccc}
1 & 0 & 1-r \\
0 & 1 & 0 \\
0 & 0 & 1
\end{array}\right)
$$

(2) By (1), we obtain a map $\mathbf{T}^{*}:[0,1]^{Y} \rightarrow[0,1]$ as

$$
\mathbf{T}^{*}(A)=\left(1-A^{*}(x)+A^{*}(z)\right) \wedge 1=(1-A(z)+A(x)) \wedge 1 .
$$

We obtain a join preserving map $\mathcal{H}_{T^{*}}(-, r): L^{X} \rightarrow L^{X}$ as follows:

$$
\mathcal{H}_{T^{*}}\left(1_{x}, r\right)(z)=\bigwedge\left\{B(z) \in L^{X} \mid B \geq 1_{x}, \mathbf{T}^{*}(B) \geq r^{*}\right\}
$$

Since $B(x)=1$ and $\mathbf{T}^{*}(B)=(1-B(z)+1) \wedge 1=1$, then $\mathcal{H}_{T^{*}}\left(1_{x}, r\right)(z)=0$.

$$
\begin{gathered}
\mathcal{H}_{T^{*}}\left(1_{z}, r\right)(y)=\bigwedge\left\{B(y) \in L^{X} \mid B \geq 1_{z}, \mathbf{T}^{*}(B) \geq r^{*}\right\}=0 \\
\mathcal{H}_{T^{*}}\left(1_{y}, r\right)(y)=\bigwedge\left\{B(y) \in L^{X} \mid B \geq 1_{y}, \mathbf{T}^{*}(B) \geq r^{*}\right\}=1 \\
\mathcal{H}_{T^{*}}\left(1_{z}, r\right)(x)=\bigwedge\left\{B(x) \in L^{X} \mid B \geq 1_{z}, \mathbf{T}^{*}(B) \geq r^{*}\right\}
\end{gathered}
$$

Since $B(z)=1$ and $\mathbf{T}^{*}(B)=1-1+B(x) \geq 1-r$, then $B(x) \geq 1-r$. We have $\mathcal{H}_{T^{*}}\left(1_{z}, r\right)(x)=1-r$.

$$
\left(\begin{array}{ccc}
\mathcal{H}_{T^{*}}\left(1_{x}, r\right)(x)=1 & \mathcal{H}_{T^{*}}\left(1_{x}, r\right)(y)=0 & \mathcal{H}_{T^{*}}\left(1_{x}, r\right)(z)=0 \\
\mathcal{H}_{T^{*}}\left(1_{y}, r\right)(x)=0 & \mathcal{H}_{T^{*}}\left(1_{y}, r\right)(y)=1 & \mathcal{H}_{T^{*}}\left(1_{y}, r\right)(z)=0 \\
\mathcal{H}_{T^{*}}\left(1_{z}, r\right)(x)=1-r & \mathcal{H}_{T^{*}}\left(1_{z}, r\right)(y)=0 & \mathcal{H}_{T^{*}}\left(1_{z}, r\right)(z)=1
\end{array}\right)
$$

For $A=\bigvee_{x \in X}\left(A(x) \odot \top_{x}\right)$, we have

$$
\begin{aligned}
& \mathcal{H}_{T^{*}}(A, r)(y)=\bigvee_{x \in X}\left(A(x) \odot \mathcal{H}_{T^{*}}\left(\top_{x}, r\right)(y)\right) . \\
& \mathcal{H}_{T^{*}}(A, r)=(A(x) \vee(A(z)-r), A(y), A(z))
\end{aligned}
$$

If $A(z)-r \leq A(x)$, then $\mathcal{H}_{T^{*}}(A, r)=A$. Thus

$$
\begin{aligned}
\mathbf{T}_{H_{T^{*}}}(A) & =\bigvee\left\{r^{*} \in L \mid \mathcal{H}_{T^{*}}(A, r)=A\right\} \\
& =(1-A(z)+A(x)) \wedge 1=\mathbf{T}^{*}(A) .
\end{aligned}
$$


Moreover, we obtain

$$
\begin{aligned}
\mathbf{T}^{* r}(A) & =\bigvee_{x \in X}\left(\mathcal{H}_{T^{*}}(A, r)(x) \rightarrow A(x)\right) \\
& =(A(x) \vee(A(z)-r)) \rightarrow A(x) \\
& =(1+r-A(z)+A(x)) \wedge 1 .
\end{aligned}
$$

For $B(x)=0.9, B(y)=0.3, B(z)=0.2, \mathbf{T}^{* 0.5}(B)=(1+0.5-B(z)+B(x)) \vee 0=$ 1.

$$
\begin{aligned}
\mathbf{T}_{T^{*}}(A) & =\bigvee\left\{1-r \in L \mid \mathbf{T}^{* r}(A)=1\right\} \\
& =(1-A(z)+A(x)) \wedge 1
\end{aligned}
$$

Hence $\mathbf{T}_{T^{*}}=\mathbf{T}_{H_{T^{*}}}=\mathbf{T}^{*}$. with

Since $R_{T^{*}}^{r}(x, y)=\mathcal{H}_{T^{*}}\left(1_{x}, r\right)(y)$, then $\mathcal{H}_{T^{*}}(A, r)(y)=\bigvee_{x \in X}\left(A(x) \odot R_{T^{*}}^{r}(x, y)\right)$

$$
R_{T^{*}}^{r}=\left(\begin{array}{ccc}
1 & 0 & 0 \\
0 & 1 & 0 \\
1-r & 0 & 1
\end{array}\right)
$$

\section{References}

[1] R. Bělohlávek,Fuzzy Relational Systems, Kluwer Academic Publishers, New York , (2002), doi: 10.1007/978-1-4615-0633-1.

[2] P. Hájek, Metamathematices of Fuzzy Logic, Kluwer Academic Publishers, Dordrecht (1998), doi: 10.1007/978-94-011-5300-3.

[3] U. Höhle, S.E. Rodabaugh,(1999), Mathematics of Fuzzy Sets: Logic, Topology, and Measure Theory, The Handbooks of Fuzzy Sets Series 3, Kluwer Academic Publishers, Boston.

[4] Fang Jinming, I-fuzzy Alexandrov topologies and specialization orders, Fuzzy Sets and Systems, 158(2007), 2359-2374, doi: 10.1016/j.fss.2007.05.001.

[5] Y.C. Kim, Alexandrov $L$-topologies and $L$-join meet approximation operators International Journal of Pure and Applied Mathematics, 91(1)(2014), 113-129 doi: 10.12732/ijpam.v9lili.12.

[6] Y.C. Kim, Join preserving maps, fuzzy preorders and Alexandrov fuzzy topologies, submit to International Journal of Pure and Applied Mathematics 
[7] J. Kortelainen, On relationships between modified sets, topological spaces and rough sets, Fuzzy Sets and Systems, 61(1994), 91-95, doi: 10.1016/0165-0114(94)90288-7.

[8] H. Lai, D. Zhang, Fuzzy preorder and fuzzy topology, Fuzzy Sets and Systems, 157 (2006), 1865-1885, doi: 10.1016/j.fss.2006.02.013.

[9] H. Lai, D. Zhang, Concept lattices of fuzzy contexts: Formal concept analysis vs. rough set theory, Int. J. Approx. Reasoning, 50 (2009), 695-707, doi: $10.1016 /$ j.ijar.2008.12.002.

[10] Z. Pawlak, Rough sets, Int. J. Comput. Inf. Sci., 11 (1982), 341-356, , doi: 10.1007/BF01001956.

[11] Z. Pawlak, Rough probability, Bull. Pol. Acad. Sci. Math., 32(1984), 607615.

[12] A. M. Radzikowska, E.E. Kerre, A comparative study of fuzy rough sets, Fuzzy Sets and Systems, 126(2002), 137-155, doi: 10.1016/so1650114(01)00032-x.

[13] S. P. Tiwari, A.K. Srivastava, Fuzzy rough sets, fuzzy preorders and fuzzy topologies, Fuzzy Sets and Systems, 210(2013), 63-68, doi: 10.1016/j.fss.2012.06.001.

[14] Y.H. She, G.J. Wang, An axiomatic approach of fuzzy rough sets based on residuated lattices, Computers and Mathematics with Applications, $\mathbf{5 8}$ (2009), 189-201, doi: 10.1016/j.camwa.2009.03.100.

[15] Zhen Ming Ma, Bao Qing Hu, Topological and lattice structures of Lfuzzy rough set determined by lower and upper sets, Information Sciences, 218(2013), 194-204, doi: 10.1016/j.ins.2012.06.029. 
